\title{
Emergency laparoscopic cryptorchidectomy for acute abdomen due to testicular torsion in a dog
}

\author{
Michal Crha ${ }^{1}$, Bart Van Goethem ${ }^{2}$, Milan Dvořák ${ }^{1}$, Alois Nečas ${ }^{1}$ \\ ${ }^{1}$ University of Veterinary and Pharmaceutical Sciences Brno, Faculty of Veterinary Medicine, \\ Small Animal Clinic, Czech Republic \\ ${ }^{2}$ Ghent University, Faculty of Veterinary Medicine, Department of Small Animal Medicine \\ and Clinical Biology, Merelbeke, Belgium
}

Received December 5, 2014

Accepted February 25, 2015

\begin{abstract}
A 7-year-old male Siberian husky was presented with signs of acute abdomen. Based on the history and diagnostic imaging findings, torsion of an undescended abdominal testis was presumed. Emergency exploratory laparoscopy was performed and confirmed spermatic cord torsion in a non-neoplastic testis. The testis was enlarged and local peritonitis was evident. Laparoscopic cryptorchidectomy was performed and the dog recovered uneventfully. Histopathology confirmed thrombosis and necrosis. Laparoscopy is not only the method of choice for elective cryptorchidectomy, but can also be used in patients with acute abdomen from testicular torsion of an undescended testis.
\end{abstract}

Torsion, undescended testis, minimally invasive surgery, peritonitis

Cryptorchidism is the most common congenital anomaly of the testes with a reported prevalence of $0.8-15 \%$ (Cox et al. 1978). Reported incidence for the Siberian husky is approximately $14 \%$ (Zhao et al. 2010). It can occur unilaterally or bilaterally and the undescended testis may be located in the abdominal cavity, inguinal canal, or in an ectopic subcutaneous location between the superficial inguinal ring and the scrotum (Evans and Christensen 1993).

When both testes are retained, the dog will be infertile due to thermal-induced testicular hypoplasia (Birchard and Nappier 2008). Cryptorchidism is also a risk factor for the development of testicular neoplasia in dogs (Yates et al. 2003). One study found the risk to develop a tumour in cryptorchid testes $13.6 \times$ as high as the risk in scrotal testes (Hayes and Pendergrass 1976). Another complication of cryptorchidism is testicular torsion. Torsion of the spermatic cord clinically manifests as an acute abdomen, and immediate therapy is indicated (Pearson and Kelly 1975).

Minimally invasive surgery has become increasingly popular in veterinary medicine and is often recommended as the preferential treatment method for elective procedures (Matyjasik et al. 2011; Zhang et al. 2014). Laparoscopic or laparoscopic-assisted cryptorchidectomy is recommended for elective removal of retained testis in dogs and cats because of the decrease in morbidity rates, improved visualization, and safety (Miller et al. 2004; Mayhew 2009; Runge et al. 2014). Descriptions of emergency laparoscopic procedures in veterinary medicine are, however, rare and the use of laparoscopic cryptorchidectomy for acute testicular torsion has not yet been described.

\section{Case description}

A 7-year-old intact male Siberian husky weighing $23 \mathrm{~kg}$ was evaluated at the Small Animal Clinic Faculty of Veterinary Medicine, University of Veterinary and Pharmaceutical Sciences Brno because of an acute onset of lethargy, moaning, and lack 
of appetite. The dog had no history of trauma and had not been treated previously for any disease.

Physical examination revealed compensatory stage of shock (hyperaemic oral mucosa, decreased capillary refill time, mild tachycardia, and severe tachypnea). Only the left testis was present in the scrotum and abdominal palpation elicited an intense pain response. Complete blood count and biochemistry tests did not reveal any abnormality.

Abdominal radiographs were taken to determine the origin of the acute abdomen (Plate V, Fig. 1A, B). A spherical soft tissue opacity mass localized cranially to the urinary bladder was detected. Abdominal ultrasound identified a mild amount of anechoic effusion in the left caudal abdominal quadrant. There was prostatomegaly $(5.6 \times 4.8 \times 7.5 \mathrm{~cm})$ with the presence of multiple small cysts $(\leq 0.7 \mathrm{~cm}$ in diameter) in the prostatic parenchyma. An oval $4.2 \times 4.0 \times 3.8 \mathrm{~cm}$ mass was found next to the bladder, with heterogeneous echogenicity yet hyperechoic to the surrounding mesenteric fat. Power Doppler mode revealed the absence of a flow signal in this mass. Since the right testis was not found, extracorporeal torsion of the right cryptorchid testis was suspected.

Given the dog's uncomfortable condition and systemic repercussions, emergency exploratory laparoscopy was performed. Cardiovascular support was instituted with lactated Ringer's solution (Hartmann's Lactated Ringer's, B. Braun, Germany) at $40 \mathrm{ml} / \mathrm{kg} / \mathrm{h}$. The dog was intravenously premedicated with midazolam $(0.1 \mathrm{mg} / \mathrm{kg}$, Dormicum, Roche, Czech Republic), medetomidine $(0.01 \mathrm{mg} / \mathrm{kg}$, Cepetor, CP-Pharma, Germany), and butorphanol (0.2 mg/kg, Butomidor, Richter-Pharma, Austria). Amoxicillin/ clavulanic acid $(20 \mathrm{mg} / \mathrm{kg}$, Augmentin $600 \mathrm{mg}$, Smith Kline Beecham Pharm, Great Britain) was administered intravenously. Anaesthesia was induced by the intravenous administration of propofol $(1 \mathrm{mg} / \mathrm{kg}$, Norofol 1\%, Norbrook Lab., Northern Ireland) and maintained by inhalation using a mixture of $\mathrm{O}_{2}$, air and isoflurane. The patient was artificially ventilated throughout the procedure with standard anaesthesia monitoring consisting of electrocardiography, pulse oximetry, esophageal body temperature measurement, end tidal $\mathrm{CO}_{2}$ and indirect blood pressure monitoring.

The patient was placed in dorsal recumbency, the urinary bladder was emptied via catheterization and the ventral abdomen was clipped and aseptically prepared for surgery. A small stab incision was made in the skin just caudal to the umbilicus, the abdominal wall was manually lifted from the organs, and the Veress needle was bluntly inserted into the peritoneal cavity. The "saline drop test" was used to verify the correct intraabdominal position of the Veress needle and pneumoperitoneum was created by insufflating $\mathrm{CO}_{2}$ to an intra-abdominal pressure of $10 \mathrm{mmHg}$. The Veress needle was replaced by a 5 -mm trocarcannula unit (EndopathExcel, Ethicon Endo-Surgery, Johnson \& Johnson, USA) in the same position. A $30^{\circ}$ forward-oblique, 5-mm telescope (Hopkins II, Karl Storz Endoscopy, Germany) was inserted through the umbilical port and used to identify the epigastric blood vessels during placement of the additional paramedian instrument ports. Two instrument ports were established $5 \mathrm{~cm}$ lateral to the umbilical port: a $5 \mathrm{~mm}$ cannula was placed on the left side and a $10 \mathrm{~mm}$ cannula on the right side. The abdomen was explored beginning with the inguinal rings: contrary to the left side, no deferent duct could be seen entering the right inguinal canal. When the haemorrhagic effusion was aspirated from the caudal abdomen, the testis was seen partially covered by omentum. The spermatic cord was rotated $360^{\circ}$ counter clockwise with the twist located $3 \mathrm{~cm}$ distal to the testis and the testis was enlarged and haemorrhagic (Plate VI, Fig. 2). Atraumatic grasping forceps were introduced through the left instrument port and the spermatic cord was clamped near the testis. A $10 \mathrm{~mm}$ vessel sealing device (LigaSure Atlas, Covidien, USA) was inserted through the right instrument port and used to coagulate and transect the spermatic cord as well as the testicular ligament. The testis was removed from the abdominal cavity using a tissue retrieval bag (Endopath Endopouch Retriever, Ethicon Endo-Surgery, Johnson \& Johnson, USA) after slightly 
enlarging the umbilical portal (Plate VI, Fig. 3). All port sites were closed in a routine manner: abdominal wall and subcutaneous tissues with polydioxanon 2/0 (PDSII, Ethicon, Johnson \& Johnson, USA), and skin with polyamide 3/0 (Ethilon II, Ethicon, Johnson \& Johnson, USA). The left scrotal testis was removed through aprescrotal approach.

Recovery was uneventful and the dog was released the day after surgery. Because signs of local peritonitis were present amoxicillin/clavulanic acid $(12.5 \mathrm{mg} / \mathrm{kg}$, Synulox, Pfizer, Czech Republic) was continued for 7 days and analgesia was obtained by meloxicam $(0.1 \mathrm{mg} / \mathrm{kg}$, Metacam, Labiana Life Sciences, Germany) for 3 days.

On the follow-up visit 14 days later the skin sutures were removed. Telephone follow-up 6 months later showed the dog to be in good health without any problems.

Histopathological examination of the testis showed that testicular as well as epididymeal tissues showed extensive interstitial haemorrhage compatible with congestion due to spermatic cord torsion. There was ischaemia-induced early necrosis of Sertoli cells and atrophy of germ cells consistent with abdominal cryptorchidism (Plate VII, Fig. 4).

\section{Discussion}

Emergency exploratory laparoscopy was performed in a dog with signs of an acute abdomen when ultrasonographic findings suggested testicular torsion. This diagnosis could quickly be confirmed, and laparoscopic cryptorchidectomy immediately relieved all signs of discomfort.

Minimally invasive cryptorchid castration (laparoscopic or laparoscopic-assisted) has been advocated as the procedure of choice for elective cryptorchidectomy since it obviates the need for "open" celiotomy and may thereby reduce postoperative discomfort and wound-related complications (Mayhew 2009). Articles on cryptorchidectomy described the incidental presence of testicular torsion (Birchard and Nappier 2008; Mayhew 2009) but specific description of emergency laparoscopy for acute abdominal pain has not been described in veterinary medicine. One article describes emergency laparoscopic exploration in a dog with persistent inguinal haemorrhage after prescrotal orchidectomy (Koenraadt et al. 2014). The authors of this article found laparoscopy in the caudal abdomen to result in superior visibility due to the absence of intestines and also noted that the presence of the pelvis created adequate working space even with low intra-abdominal pressures. The latter being of benefit to cardiovascular unstable patients. In human medicine emergency laparoscopic exploration is more frequently used to identify the conditions underlying acute abdominal pain (Agresta et al. 2006). In stable patients with acute abdominal pain, noninvasive diagnostics are first fully exhausted, but when routine diagnostic procedures fail to produce results, laparoscopy is advocated (Sauerland et al. 2006). A protocol much like this was followed for the dog in this report where bloodwork, abdominal radiography, and ultrasonography preceded the laparoscopic exploration.

Because retained testes can be unilateral or bilateral, are usually small and atrophied, and vary in location, diagnosis can be challenging in some animals (Birchard and Nappier 2008). Animals with testicular torsion usually present with acute abdominal pain and lethargy as evident in this case. But diagnosis of torsion of non-neoplastic retained testis is difficult because it mimics other acute abdominal emergencies (Hecht et al. 2004; Beal 2005). Abdominal radiography may yield inconsistent results in terms of delineation of the testis (Beal 2005). Due to the increase in size of the abdominal testis in this case it was picked up on plain radiography as a soft tissue opacity cranially to the urinary bladder. Still, a paraprostatic cyst, enlarged lymph nodes, intestinal tumor or other mass involving an organ in this localization are all valid differentials. Ultrasonography is highly sensitive for determining the location of undescended testis: $96.6 \%$ and $100 \%$ for abdominal and inguinal retained testes, respectively (Felumlee et al. 2012). Furthermore the lack of 
vascularization detected on colour Doppler mode confirmed the presence of testicular torsion (Hecht et al. 2004).

Predominant features of acute torsion $(<6 \mathrm{~h})$ are testicular enlargement and hypoechogenicity of the parenchyma, mixed echogenicity is typical for the subacute fase (1-10 days) and in the chronic phase ( $>10$ days) the testis appears small and hypoechoic (Hecht et al. 2004). Although hyperechogenicity, as seen in the present case, is considered rare (Johnston et al. 1991) it has been described before in a case of acute torsion (Hecht et al. 2004). Mixed echogenicity is also described with neoplastic changes of an intraabdominal testis (Miyabashi et al. 1990).

Cryptorchid testes are predisposed to the development of neoplasia (Birchard and Nappier 2008). The increase in size and weight of neoplastic testis increases the risk of rotation of the pendulous testicle (Pearson and Kelly 1975; Miyabashi et al. 1990). Most reports therefore describe torsion as a sequel of neoplastic transformation. Interestingly, spermatic cord torsion in neoplastic testis is more often an incidental finding and congestive or necrotic changes are seldom observed. Presumably, this is due to tumourinduced increase of blood supply allowing torsion without thrombosis (Restucci et al. 2003).

Non-neoplastic ectopic abdominal testes will atrophy due to thermal suppression of sperm production (Birchard and Nappier 2008). This makes them less prone to torsion resulting in sparse descriptions of testicular torsion of non-neoplastic testis (White and Johnson 1955; Eskew and Kuhn 1961; Naylor and Thompson 1979; MacDonald et al. 1961; Salmon 1970; Turner 1970). But when torsion of a non-neoplastic/atrophied testis does occur this will quickly result in venous infarction inducing both testicular and epididymal haemorrhage. Testicular size increases and mechanical forces on the spermatic cord promote further constriction of blood vessels. When the arterial blood supply is discontinued, testicular necrosis quickly follows (Foster 2012). The testis found in this case was considered enlarged instead of atrophied, and signs of early necrosis were evident on histopathology fitting the above hypothesis.

The twisted testis in this case measured approximately $4 \mathrm{~cm}$ in diameter. One of the port sites therefore needed to be enlarged to allow for the removal of this testis. The authors preferred to enlarge the $5 \mathrm{~mm}$ umbilical camera portal rather than the $10 \mathrm{~mm}$ right paramedian instrument port, hypothesizing the latter would create more abdominal wall muscle damage and potentially lead to complications in surgical wound healing (Mayhew 2009). An alternative approach that avoids the need for later enlargement of the portal sites would be to use a $3 \mathrm{~cm}$ single incision laparoscopic surgery (SILS) cannula (Runge et al. 2014). The resected testis is usually easily removed from the abdomen via $10 \mathrm{~mm}$ paramedian (working) port incision. If the testes are very large $(8$ to $10 \mathrm{~cm})$, the laparoscopic approach may be less practical because a large incision will be required to retrieve the testis from the abdomen (Mayhew 2009).

Based on size and echogenicity the testis in this case was at the time of surgery considered potentially neoplastic. A specimen retrieval bag was therefore used, because it limits the spread of neoplastic cells in the abdomen and port-site metastasis when neoplastic tissue is being pulled through an instrument port (Mayhew 2009). In the authors' experience, emergency laparoscopic castration of an acutely twisted abdominally retained testis was a fast, safe, and relatively simple procedure. It could be considered standard treatment approach for testes not larger than $5 \mathrm{~cm}$.

\section{Acknowledgment}

Laparoscopic instruments were supported by IGA VFU Brno (44/2013/FVL).

The authors thank Assoc. Prof. Misa Skoric of the Department of Pathological Morphology and Parasitology, FVM UVPS Brno, for providing the histopathological illustration. 


\section{References}

Agresta F, Ciardo LF, Mazzarolo G, Michelet I, Oris G, Trentin G, Bedin N 2006: Peritonitis: laparoscopic approach. World J Emerg Surg 1: 9

Beal MW 2005: Approach to the acute abdomen. Vet Clin North Am Small Anim Pract 35: 375-396

Birchard SJ, Nappier M 2008: Cryptorchidism. Compend Contin Educ Vet 30: 325-337

Cox VS, Wallace LJ, Jessen CR 1978: An anatomic and genetic study of canine cryptorchidism. Teratology 18: 233-240

Eskew NE, Kuhn EF 1961: Case report: abdominal pain due to torsion of a retained testicle. Vet Med 56: $212-213$

Evans HE, Christensen GC 1993: The urogenital system. In: Evans HE (Ed.): Miller's Anatomy of the Dog, $3^{\text {rd }}$ edn. Saunders, Philadelphia, pp. 508-513

Felumlee AE, Reichle JK, Hecht S, Penninck D, Zekas L, Yeager AD, Goggin JM, Lowry J 2012: Use of ultrasound to locate retained testes in dogs and cats. Vet Radiol Ultrasound 53: 581-585

Foster RA 2012: Common lesions in the male reproductive tract of cats and dogs. Vet Clin North Am Small Anim Pract 42: 527-545

Hayes HM, Pendergrass TW 1976: Canine testicular tumors: epidemiological features of 410 dogs. Int J Canc 18: $482-487$

Hecht S, King R, Tidwell AS, Gorman SC 2004: Ultrasound diagnosis: intraabdominal torsion of a non-neoplastic testicle in a cryptorchid dog. Vet Radiol Ultrasound 45: 58-61

Johnston GR, Feeney DA, Rivers B, Walter PA 1991: Diagnostic imaging of the male canine reproductive organs. Methods and limitations. Vet Clin North Am Small Anim Pract 21: 553-589

Koenraadt A, Stegen L, Bosmans T, Van Goethem B 2014: Laparoscopic treatment of persistent inguinal haemorrhage after prescrotal orchiectomy in a dog. J Small Anim Pract 55: 427-430

Matyjasik H, Adamiak Z, Pesta W, Zhalniarovich Y 2011: Laparoscopic procedures in dogs and cats. Pol J Vet Sci 14: 305-316

Mayhew P 2009: Laparoscopic and laparoscopic-assisted cryptorchidectomy in dogs and cats. Compend Contin Educ Vet 31: 274-281

MacDonald DS, Devereux RJ, Bartolf F 1961: Torsion of an ectopic testicle simulating a foreign body in the intestine. Can Vet J 2: 117

Miller NA, Van Lue SJ, Rawlings CA 2004: Use of laparoscopic-assisted cryptorchidectomy in dogs and cats. J Am Vet Med Assoc 224: 875-878

Miyabashi T, Biller DS, Cooley AJ 1990: Ultrasonographic appearance of torsion of a testicular seminoma in a cryptorchid dog. J Small Anim Pract 31: 401-403

Naylor RW, Thompson SMR 1979: Intra-abdominal testicular torsion - a report of two cases. J Am Anim Hosp Assoc 15: 763-766

Pearson H, Kelly DF 1975: Testicular torsion in the dog: a review of 13 cases. Vet Rec 97: 200-204

Restucci B, Maiolino P, Paciello O, Martano M, De Vico G, Papparella S 2003: Evaluation of angiogenesis in canine seminomas by quantitative immunohistochemistry. J Comp Pathol 128: 252-259

Runge JJ, Mayhew PD, Case JB, Singh A, Mayhew KN, Culp WT 2014: Single-port laparoscopic cryptorchidectomy in dogs and cats: 25 cases (2009-2014). J Am Vet Med Assoc 245: 1258-1265

Salmon RD 1970: Letter to the editor. J Small Anim Pract 11: 703

Sauerland S, Agresta F, Bergamaschi R, Borzellino G, Budzynski A, Champault G, Fingerhut A, Isla A, Johansson M, Lundorff P, Navez B, Saad S, Neugebauer EA 2006: Laparoscopy for abdominal emergencies: evidence-based guidelines of the European Association for Endoscopic Surgery. Surg Endo 20: 14-29

Turner T 1970: Torsion of the retained testicle in the dog. J Small Anim Pract 11: 436-437

White PT, Johnson P 1955: Strangulated testicle of a cryptorchid (dog). J Am Vet Med Assoc 126: 312

Yates D, Hayes G, Heffernan M, Beynon R 2003: Incidence of cryptorchidism in dogs and cats. Vet Rec 152: 502-504

Zhao X, Du ZQ, Rothschild MF 2010: An association study of 20 candidate genes with cryptorchidism in Siberian Husky dogs. J Anim Breed Genet 127: 327-331

Zhang H, Zhou Z, Zhang J, Zhang S, Wang H 2014: Laparoscopic cystostomy in pigs: Technique and comparison with traditional open cystostomy for surgical stress. Acta Vet Brno 83: 385-391 
Plate V

Crha M. et al.: Emergency laparoscopic ... pp. 167-171
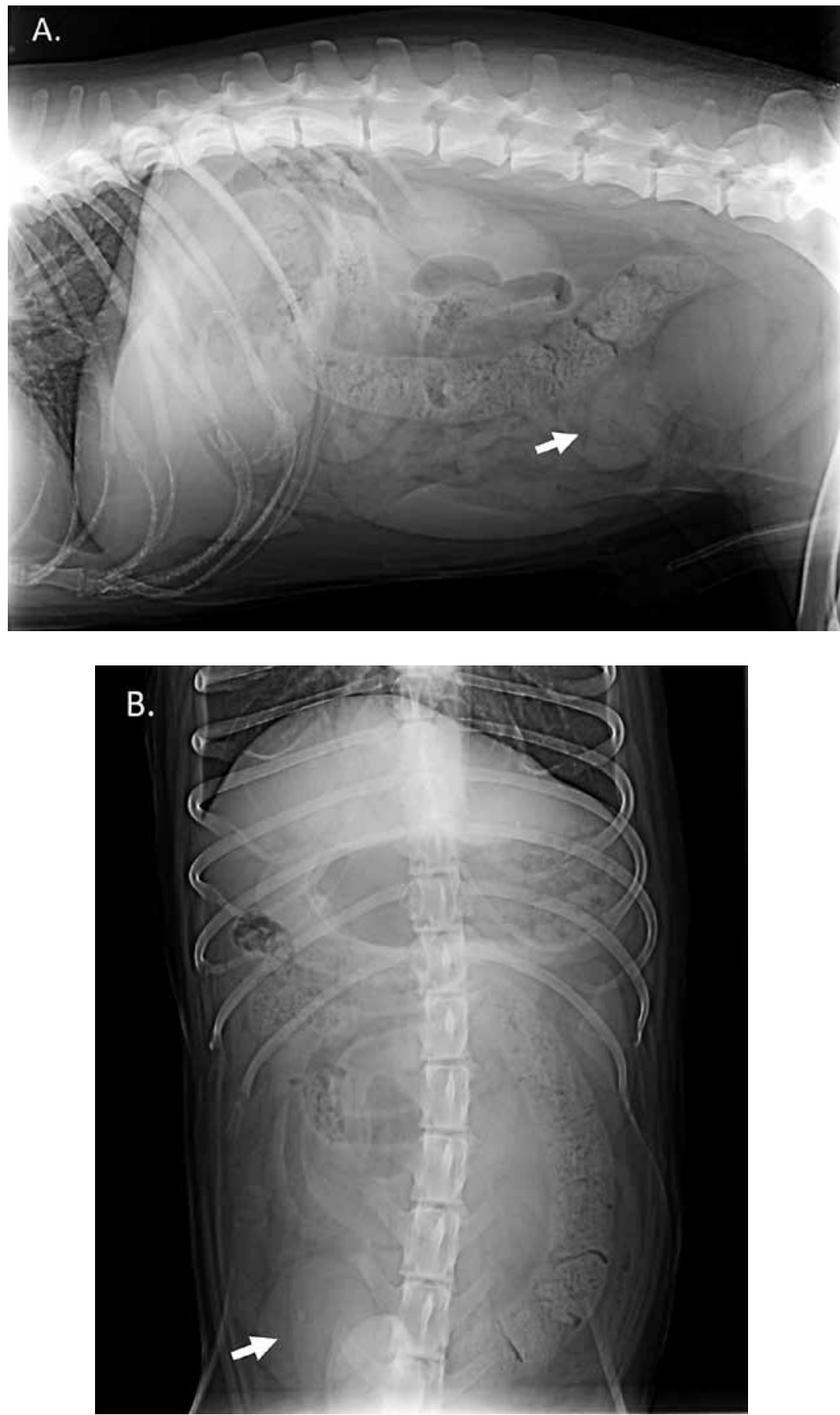

Fig. 1. Abdominal radiographs (A - latero-lateral and B - ventro-dorsal) revealing a soft tissue opacity mass (arrow) cranial to the urinary bladder 


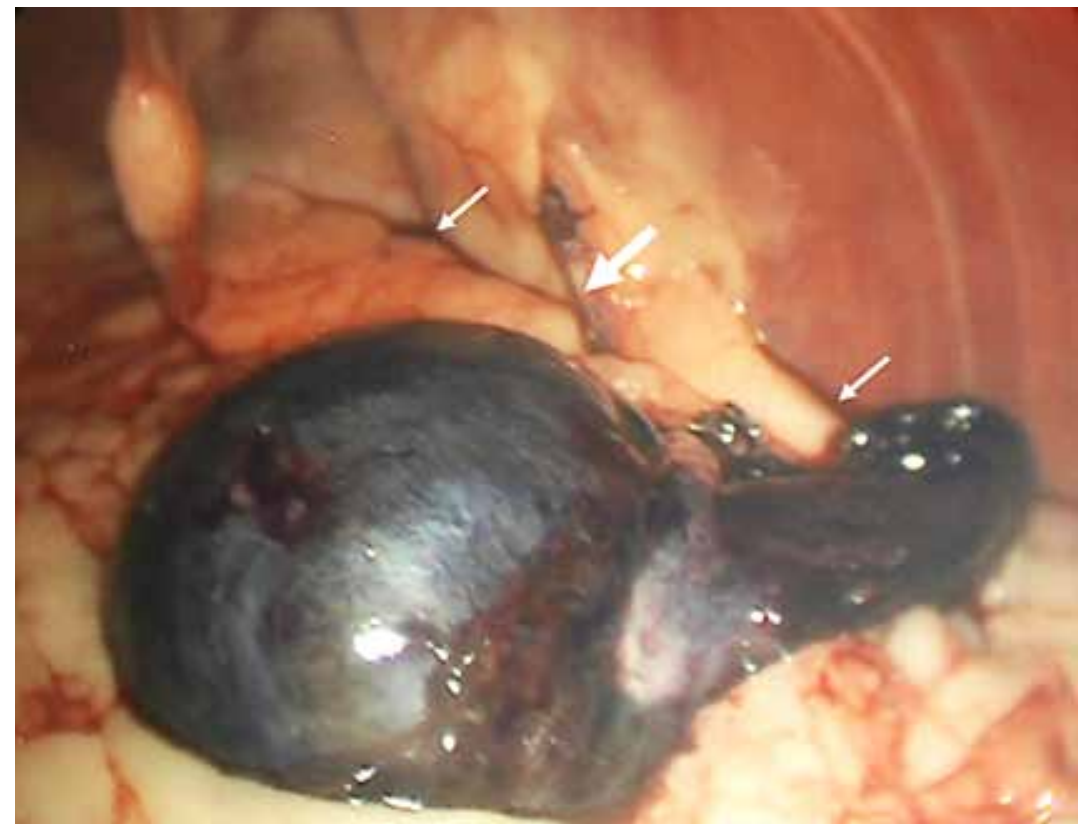

Fig. 2. The right testis was enlarged and congested due to spermatic cord torsion. Note the presence of free fluid in the abdomen (small arrows) and haemorrhagic changes in the gubernaculum (broad arrow)

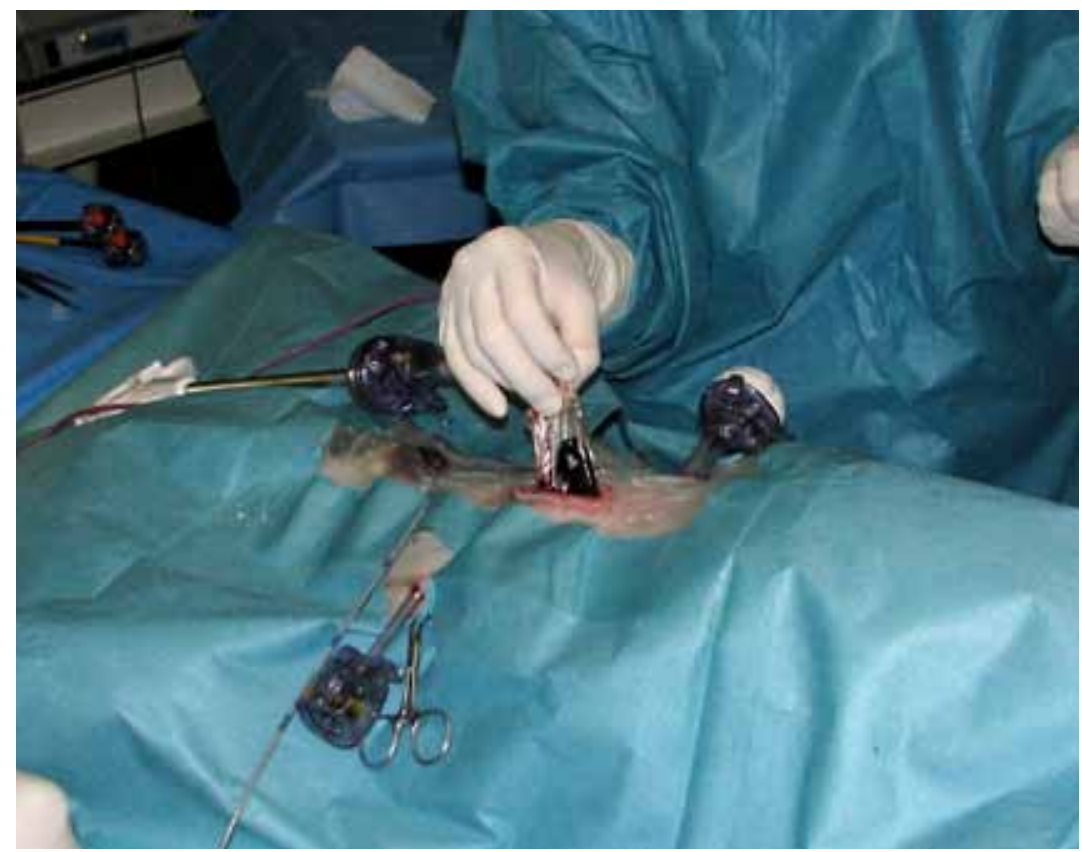

Fig. 3. The transected testis was removed from the abdominal cavity in a laparoscopic tissue retrieval bag after enlarging the umbilical port site (head is to the left). 


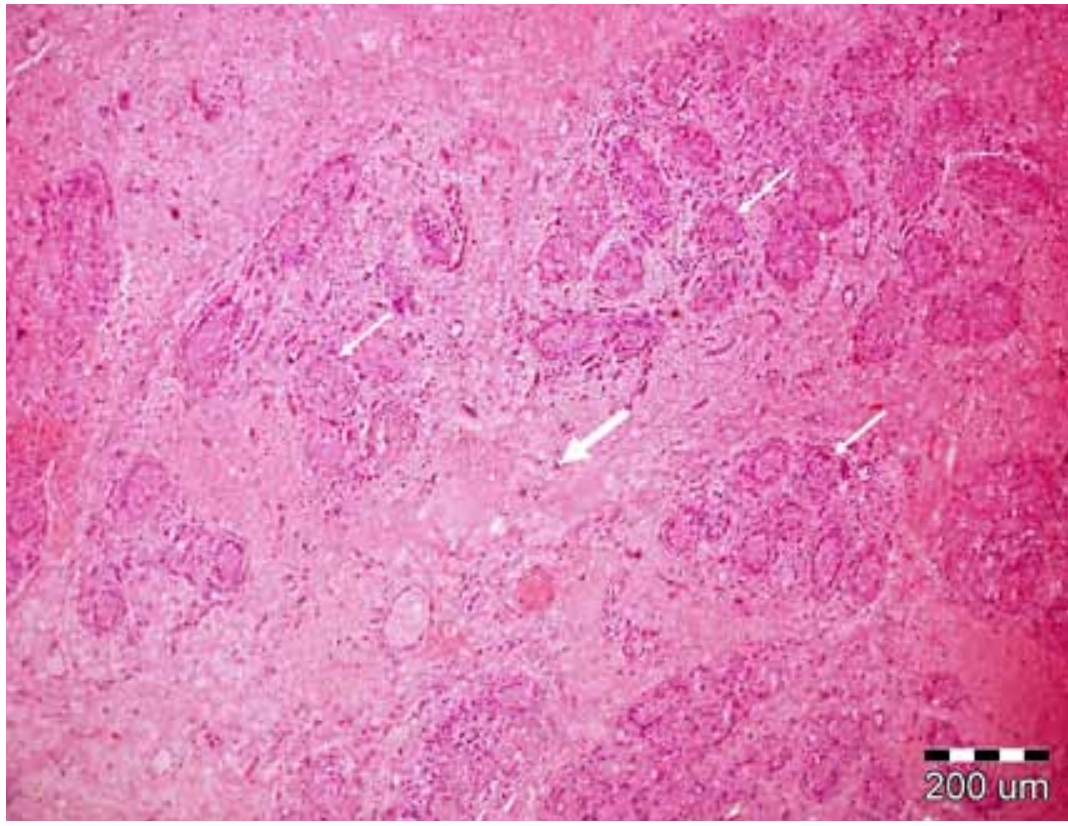

Fig. 4. Diffuse parenchymal haemorrhagic necrosis of the testis (broad arrow) with visible outlines of tubular structures (small arrows), HE stain, magnification $\times 100$ 